\title{
Entropy and Entropic Forces to Model Biological Fluids ${ }^{+}$
}

\author{
Rafael M Gutierrez, Chandrashekhar U Murade, Jianfeng Guo and George Shubeita \\ Science Division, New York University, Abu Dhabi, United Arab Emirates \\ + Presented at the Entropy 2021: The Scientific Tool of the 21st Century, 5-7 May 2021; Available online: \\ https://sciforum.net/conference/Entropy2021/.
}

Published: 5 May 2021

Biological systems tend to exhibit common organizational patterns despite they diversity and different spatial and temporal scales. Living cells are complex systems that may be characterized by fluids crowded by hundreds of different elements in particular by a high density of polymers; they are an excellent and challenging laboratory to study exotic emerging physical phenomena where entropic forces emerge from organization processes of many-body interactions. There may be many entropic forces emerging in a biological fluid but most of them are consequences or can be reduced to the crowding and exclusion volume effects. Since entropic forces are emergent phenomena resulting from the tendency of a thermodynamic system to maximize its entropy, the macroscopic variables describing the system tend to evolve from one state to another state that is statistically more probable. If an external force exerted on the system point in the direction of decreasing its entropy while the entropic forces generated by the system point in the direction of increasing its entropy, when the system reaches its maximum entropy, the entropic force becomes zero. Therefore, the competition between entropic and physical forces may generate complex behaviors like phase transitions that living cells may use to accomplish their functions. In the era of the big data, when biological information abounds but general principles and precise understanding of the microscopic interactions scarce, the entropy methods may offer significant information including statements of the physical interactions between the diversity of constituent elements, inferred only from experimental data. In this work we develop a model of a biological fluid involving the competition of entropic and physical forces that living cells seem to use to accomplish their functions as has been recently started to be understood. The target audience for this article are interdisciplinary researchers in complex systems, particularly in biophysics modeling.

(C) 2021 by the authors. Licensee MDPI, Basel, Switzerland. This article is an open access article distributed under the terms and conditions of the Creative Commons Attribution (CC BY) license (http://creativecommons.org/licenses/by/4.0/). 